\title{
Resultados de la prueba inmunoquímica fecal en un programa de escrutinio para cáncer colorrectal en México
}

\author{
Results of the fecal immunochemical test in a colorectal cancer screening program in \\ Mexico
}

\begin{abstract}
Shareni Gálvez-Ríos', Sergio Sobrino-Cossío², Ana Siu ${ }^{3}$, Marlene Chaurand", Miguel Abdo-Francis 5 , Aurelio López-Colombo ${ }^{6}$, Eduardo Fenocchi ${ }^{7}$,Elymir Galvis-García ${ }^{2,8}$, Óscar Teramoto-Matsubara ${ }^{9}$, Arturo Meixueiro-Daza', Peter Grube-Pagola1, Paulo C. Gómez-Castaños ${ }^{1}$, Olivia Rascón-Sosa', Xaira J. Rivera-Gutiérrez', Orestes Cobos-Quevedo ${ }^{1}$, Enrique Pérez-Luna ${ }^{1}$ y José M. Remes-Troche ${ }^{1 *}$ ${ }^{1}$ Laboratorio de Fisiología, Instituto de Investigaciones Médico-Biológicas, Universidad Veracruzana, Veracruz; ${ }^{2}$ Departamento de Endoscopia, Hospital Ángeles del Pedregal, Ciudad de México; ${ }^{3}$ Departamento de Endoscopia, Clínica Médica Siu, Tuxtla Gutiérrez, Chiapas; ${ }^{4}$ Departamento de Endoscopia, Hospital Regional de Alta Especialidad de la Península de Yucatán, Yucatán; ${ }^{5}$ Departamento de Endoscopia, Hospital Ángeles Acoxpa, Ciudad de México; ${ }^{6}$ Dirección de Enseñanza, Hospital de Especialidades, Centro Médico Nacional Manuel Ávila Camacho, Instituto Mexicano del Seguro Social, Puebla de Zaragoza, Puebla. México; ${ }^{7}$ Departamento de Endoscopia, Centro de Cáncer Digestivo, Instituto Nacional del Cáncer, Montevideo, Uruguay; ${ }^{8}$ Servicio de Endoscopia, Hospital General de México Dr. Eduardo Liceaga, Ciudad de México; ${ }^{9}$ Departamento de Endoscopia, Centro Médico ABC, Ciudad de México. México
\end{abstract}

\section{Resumen}

Antecedentes: El cáncer colorrectal (CCR) es una de las cinco primeras causas de morbimortalidad por cáncer en nuestro país y en todo el mundo. La prueba inmunoquímica fecal (FIT, fecal immunochemical test) es una herramienta de tamizaje no invasiva que permite seleccionar a los sujetos con mayor probabilidad de lesión premaligna en la colonoscopia. Objetivo: Reportar los resultados del programa de escrutinio para CCR mediante FIT en población abierta en México. Método: Estudio multicéntrico nacional en población abierta mayor de 50 años a través de medios de difusión masiva para participar en un programa de escrutinio de CCR. Se utilizó FIT cuantitativa específica para detectar hemoglobina humana con un punto de corte de $100 \mathrm{ng} / \mathrm{ml}$ (prueba positiva). Se realizó colonoscopia a los positivos. Se tomaron biopsias dirigidas de las lesiones premalignas/cáncer para análisis histopatológico. Resultados: Se procesaron 751 FIT, de las cuales 51 (6.8\%) fueron positivas, con una tasa de 15.9 lesiones premalignas por cada 1,000 sujetos evaluados, y 1.3 pacientes con CCR por cada 1,000 pacientes. Conclusiones: Nuestro estudio concuerda con lo reportado en la literatura mundial, apoyando así la iniciativa de fomentar el establecimiento de un tamizaje formal y estandarizado dentro del sector de salud pública.

Palabras clave: Cáncer colorrectal. Escrutinio. Prueba inmunoquímica fecal.

\section{Abstract}

Background: Colorectal cancer $(C R C)$ is one of the main five causes of morbidity and mortality by oncologic diseases in our country and worldwide. Recently, fecal immunochemical test (FIT) has proven to be a noninvasive screening test that allows

Fecha de recepción: 14-05-2019 Fecha de aceptación: 05-06-2020 C.P. 91700 , Veracruz, Ver., México E-mail: jose.remes.troche@gmail.com DOI: $10.24875 / C I R U .20001299$

Cir Cir. 2020;88(5):635-642 Contents available at PubMed www.cirugiaycirujanos.com 0009-7411/@ 2020 Academia Mexicana de Cirugía. Publicado por Permanyer. Este es un artículo open access bajo la licencia CC BY-NC-ND (http://creativecommons.org/licenses/by-nc-nd/4.0/).
} 
to select patients most likely to have a pre-malign lesion in order to perform a colonoscopy. Objective: To report the findings of a CRC screening program using FIT in our country population. Method: A multicentric study was performed, by inviting open population older than 50 years to participate in a CRC screening. Quantitative FIT specific for human hemoglobin was used, with a cut point of $100 \mathrm{ng} / \mathrm{ml}$ or higher to consider as positive. Those patients with positive results were asked to undergo a colonoscopy. In the cases where polypoid lesions were found, biopsies were performed. Results: In total, 751 FIT were processed, and 51 (6.8) of those were positive, with a rate of 15.9 premalign lesions for 1,000 individuals, and 1.3 patients with CRC for every 1,000. Conclusions: The present study matches worldwide reports, supporting the initiative of establishing a formal and standardized CRC screening program in the public health sector.

Key words: Colorectal cancer. Screening. Fecal immunochemical test.

\section{Introducción}

El cáncer colorrectal (CCR) es la tercera causa de morbimortalidad oncológica en el mundo, tanto en hombres como en mujeres. De acuerdo con el registro GLOBOCAN, en 2012 la incidencia estimada fue de 17.2 nuevos casos por cada 100,000 habitantes, con una tasa de mortalidad de $8.4 / 100,000$, la cual se incrementó al $9.2 \%$ en $2018^{1-3}$.

En el continente americano, la incidencia estimada es de 246,000 nuevos casos, con una mortalidad de aproximadamente 112,000 personas cada año. Las tasas de incidencia y mortalidad en América Latina y el Caribe son menores que en los Estados Unidos de América y Canadá, con excepción de las de Uruguay, Argentina, Barbados y Trinidad y Tobago, cuyas cifras son mayores que en el resto de América Latina y similares a las de Norteamérica. En México, el CCR ocupa el tercer lugar en incidencia entre las enfermedades oncológicas en ambos sexos, y el quinto lugar en mortalidad en hombres y el sexto en mujeres ${ }^{1,2}$.

La incidencia y la mortalidad del cáncer están creciendo rápidamente en todo el mundo. De acuerdo con las estimaciones de la Organización Mundial de la Salud (OMS) en 2015, CCR representó la tercera causa de muerte en general y la segunda causa de muerte por cáncer en los adultos mayores de 70 años. El registro GLOBOCAN estimó para el año 2018 la detección de 18.1 millones de nuevos casos y 9,6 millones de muertes-cáncer en el mundo. El continente americano representa el $21 \%$ de la incidencia y el $14.4 \%$ de la mortalidad por cáncer ${ }^{3,4}$.

Las diferencias en los patrones de mortalidad pueden explicarse por las distintas infraestructuras de salud, el tratamiento y el acceso a la atención médica, y los mayores costos del diagnóstico ${ }^{4}$. Los países en transición hacia niveles más altos de desarrollo humano, como los de América Central y del Sur, y los de Europa del Este, han mostrado un incremento en la incidencia de CCR.
Los países de América Central y América del Sur (incluida Cuba) están experimentando rápidos cambios sociodemográficos y epidemiológicos, y la naturaleza de los problemas de salud está pasando por una transición de enfermedades infecciosas a enfermedades crónicas, incluido el cáncer ${ }^{5}$. Recientes estimaciones indican que el $15 \%$ de los cánceres de colon y el $8 \%$ de los cánceres rectales ocurrirán en América Latina (incluyendo el Caribe) $)^{4,5}$.

El CCR se puede dividir en hereditario y esporádico. En la mayor parte de los casos presenta un desarrollo progresivo a partir de una lesión premaligna (adenomatosa), que después de un período de alrededor de 10 años da origen a una lesión maligna, lo que se conoce como «secuencia adenoma-carcinoma» ${ }^{6,7}$. Por ello, al presentar un «período de ventana», es posible detectar y tratar oportunamente lesiones premalignas e incluso cánceres en etapas tempranas, lo cual repercute en la expectativa de vida y en los gastos del sector salud de manera positiva.

Es importante recordar que el CCR solo presenta síntomas en las etapas avanzadas de la enfermedad. En general, el paciente acude a la consulta ante la presencia de datos de alarma (diagnóstico clínico usual). La magnitud de la hemorragia es variable y es una consecuencia del traumatismo del tejido neoplásico debido al paso de la materia fecal a través del colon; es decir, es un signo de alarma asociado con el crecimiento tumoral. Es en dicho problema donde reside la importancia de establecer programas de escrutinio para población general, en individuos asintomáticos y mayores de 50 años. Las pruebas utilizadas en programas de tamizaje, idealmente, deberían tener un bajo costo, buenas sensibilidad y especificidad, ser fáciles de aplicar, no invasivos y tener buena adherencia ${ }^{8-10}$.

Entre las pruebas utilizadas para el tamizaje de CCR en diferentes países se encuentran los estudios de sangre oculta en heces, la rectosigmoidoscopia flexible, la colonoscopia y la colonografía por tomografía computarizada. Sin embargo, solo los 
exámenes de sangre oculta en heces por prueba de guayaco y la sigmoidoscopia flexible han demostrado disminuir la incidencia y la mortalidad de este tipo de cáncer en estudios controlados y aleatorizados ${ }^{11-16}$.

Los estudios menos invasivos, de menor costo, y por lo tanto de mayor aceptación entre la población, son los de sangre oculta en heces. El avance más reciente para evaluar la sangre oculta en heces es la prueba inmunoquímica fecal (FIT, fecal immunochemical test). Su predecesora, la prueba de guayaco, se basa en la detección de peroxidasa en sangre, por lo que también puede reaccionar de manera inespecífica a la peroxidasa presente en alimentos como carnes rojas, frutas y algunos vegetales, y además puede dar falsos negativos si el paciente se encuentra consumiendo altas dosis de vitamina $\mathrm{C}$, la cual bloquea la reacción de la peroxidasa. Por otra parte, la FIT se basa en una reacción antígeno-anticuerpo específica para detectar globina humana, y cuenta con la ventaja de ser más específica para el sangrado digestivo bajo, ya que la globina proveniente del tubo digestivo alto es degradada por enzimas digestivas antes de llegar al tracto inferior ${ }^{13}$.

Existen dos tipos de FIT: cualitativa y cuantitativa. La primera arroja un resultado positivo o negativo, mientras que la segunda indica la concentración de globina. La FIT cuantitativa es de elección para el tamizaje de poblaciones, ya que brinda la ventaja de seleccionar un punto de corte para positividad de la prueba dependiendo de las características del propio equipo (recomendaciones del proveedor) y del nivel de riesgo de la población que se está sometiendo a escrutinio, y por tanto se pueden seleccionar de manera más confiable aquellos pacientes que ameritan estudio de colonoscopia; es decir, permite al clínico seleccionar un balance entre sensibilidad y especificidad dependiendo de la población estudiada y de los recursos endoscópicos con los que se cuenta ${ }^{13-16}$.

Actualmente no existe una recomendación universal para el escrutinio del CCR, pero la mayoría de las guías y de los consensos recomiendan iniciarlo a partir de los 50 años en personas con riesgo bajo y a los 40 años en personas con riesgo elevado. En todo el mundo, el estudio más recomendado y utilizado es la colonoscopia; sin embargo, es importante destacar que esto es más frecuente en los países desarrollados, mientras que en los países en vías de desarrollo la recomendación más frecuente para el tamizaje es la identificación de sangre oculta en heces. En México, el programa para escrutinio del CCR basado en sangre oculta en heces va dirigido a personas con riesgo bajo, mayores de 50 años, y se recomienda realizar anualmente la prueba (de guayaco o inmunoquímica); en caso de que dicha prueba se encuentre alterada, se deberá realizar colonoscopia ${ }^{14-16}$.

El objetivo del presente estudio es reportar los hallazgos de un programa de escrutinio para CCR mediante FIT en población abierta en México, con el fin de determinar la prevalencia de lesiones tempranas asociadas a CCR.

\section{Método}

Se realizó un estudio transversal, prospectivo, descriptivo y multicéntrico, con la participación de los Estados de México, Veracruz, Chiapas, Yucatán y Puebla, iniciado en mayo de 2015 y finalizado en enero de 2016. Mediante convocatoria en centros de salud de diferentes niveles y a través de medios de difusión masiva, se invitó a la población abierta mayor de 50 años a participar en un programa de escrutinio para cáncer de colon. Se excluyó a los pacientes con antecedente personal de CCR, enfermedad inflamatoria intestinal, melena, hemorroides con hemorragia activa o datos de alarma, como pérdida de peso injustificada. Se recabaron los datos personales del paciente, se otorgó un número de identificación con el cual se rotuló el dispositivo para la toma de la muestra de heces, y se explicó el proceso para la recolección de la muestra en casa. Una vez recabada la muestra, fue almacenada en refrigeración para su posterior traslado a un centro de lectura en la Ciudad de México, donde se llevó a cabo el análisis de las muestras de manera centralizada. Se realizó una FIT cuantitativa específica para hemoglobina $(\mathrm{Hb})$ humana, la cual se procesó mediante el método de prueba automatizado OC FIT$\mathrm{CHEK}^{\circledast}$ (Polymedco-Endomedica S.A. de C.V.) ${ }^{17,18}$. El punto de corte se estableció en $100 \mathrm{ng} / \mathrm{ml}$ para considerar la prueba como positiva, de acuerdo con las recomendaciones del proveedor. Los pacientes con resultados positivos fueron contactados para realizarles una colonoscopia, la cual se realizó previo consentimiento informado y tras preparación intestinal.

En los casos en que durante la colonoscopia se identificaron pólipos, o con sospecha de malignidad, se recolectaron biopsias para su posterior análisis histopatológico.

El protocolo fue aprobado por el Comité de Ética Institucional y se siguió cumpliendo los derechos de los pacientes de acuerdo con la Declaración de Helsinki. No hubo ningún apoyo económico ni 


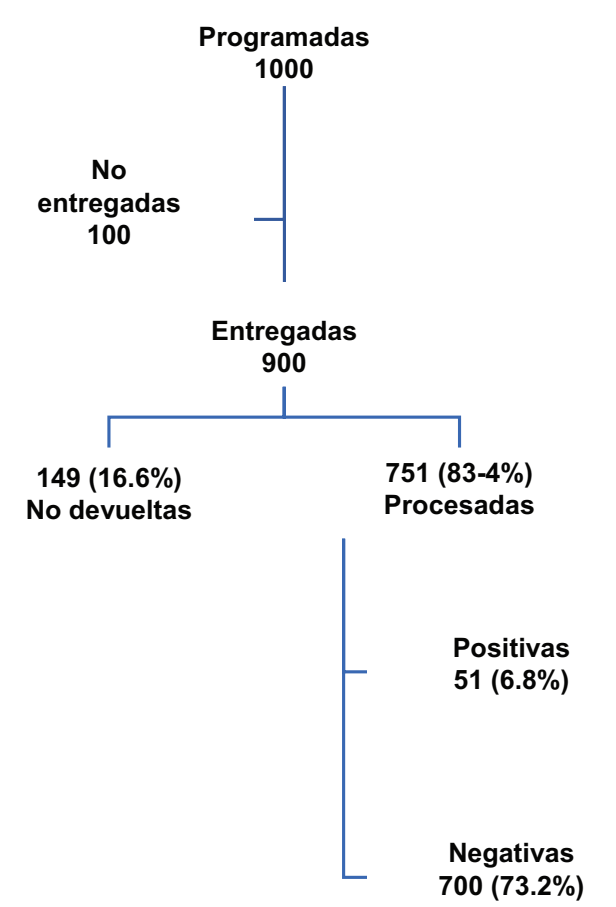

Figura 1. Proyección y procesamiento después de la aplicación de 1000 pruebas inmunoquímicas fecales cuantitativas para la detección de sangre oculta en heces.

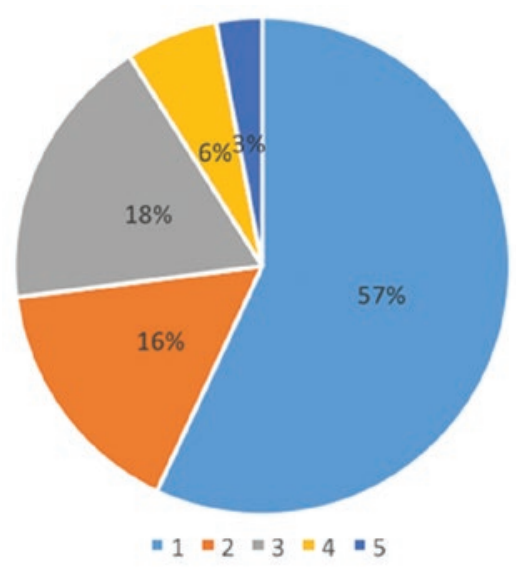

Figura 2. Porcentaje de muestras aplicadas por cada Estado de la República. 1: Veracruz, Veracruz. 2: Ciudad de México. 3: Tuxtla Gutiérrez, Chiapas. 4: Mérida, Yucatán. 5: Puebla, Puebla.

remuneración para los sujetos de estudio, quienes aceptaron participar de forma voluntaria.

Para el análisis se utilizó estadística descriptiva y para la comparación de variables entre grupos con distribución anormal se empleó la prueba $U$ de Mann Withney. Se usó el paquete estadístico SPSS versión 11.00 y se estableció significancia estadística cuando p fue $<0.05$.
Tabla 1. Pruebas inmunoquímicas de sangre oculta en heces (FIT) y colonoscopias realizadasM

\begin{tabular}{lccc}
\hline Estado & $\begin{array}{c}\text { FIT realizadas } \\
\mathbf{n}(\%)\end{array}$ & $\begin{array}{c}\text { FIT positivas } \\
\mathbf{N}(\%)\end{array}$ & $\begin{array}{c}\text { Colonoscopias } \\
\text { realizadas }\end{array}$ \\
\hline $\begin{array}{l}\text { Tuxtla Gutiérrez, } \\
\text { Chiapas }\end{array}$ & $133(18)$ & $12(9)$ & 12 \\
$\begin{array}{l}\text { Ciudad de } \\
\text { México }\end{array}$ & $118(16)$ & $12(10.2)$ & 8 \\
$\begin{array}{l}\text { Veracruz, } \\
\text { Veracruz }\end{array}$ & $430(57)$ & $25(5.8)$ & 18 \\
$\begin{array}{l}\text { Puebla, Puebla } \\
\text { Mérida, }\end{array}$ & $22(3)$ & $0(0)$ & 0 \\
Yucatán & $48(6)$ & $2(4.2)$ & 2 \\
Total & 751 & $51(6.8)$ & $40(78.4 \%)$ \\
\hline
\end{tabular}

\section{Resultados}

Se proyectó el uso de 1,000 FIT cuantitativas, de las cuales $900(90 \%)$ fueron aplicadas y finalmente $751(75 \%)$ fueron devueltas y procesadas (Fig. 1). De las pruebas procesadas, 51 resultaron positivas ( $\geq 100 \mathrm{ng} / \mathrm{ml}$ ), es decir, el $6.8 \%$ (intervalo de confianza del 95\%: 5.5-8.2), con variaciones según las regiones, desde el $4.2 \%$ en Yucatán hasta el $10.2 \%$ en Ciudad de México, dependiendo también de la cantidad de muestras totales proporcionadas por cada región (Fig. 2 y Tabla 1). El 59.5\% del total de los sujetos incluidos ( $n=447)$ fueron mujeres, y la media de edad fue de 57 años, con un promedio de índice de masa corporal de $27 \mathrm{~kg} / \mathrm{m}^{2}$ (Tabla 2).

Se contactó a los 51 pacientes con resultado positivo y se logró el seguimiento de 40 (78.4\%) de ellos por medio de colonoscopia. Del los 40 estudios endoscópicos realizados, en cinco $(12.5 \%)$ no hubo hallazgo de lesiones colorrectales. Para el análisis se dividieron en dos grupos de acuerdo con el resultado de la colonoscopia: pacientes con hallazgo de lesiones no neoplásicas y pacientes con lesiones neoplásicas (pólipos sin y con potencial de malignidad, y cáncer ya establecido).

En 20 pacientes se encontraron únicamente hallazgos no neoplásicos, como proctitis (2), enfermedad hemorroidal (12), enfermedad diverticular (5), colitis ulcerosa crónica inespecífica (2), angiodisplasia (2) y colitis amebiana (2). En 15 pacientes hubo hallazgos neoplásicos, como pólipos sin atipias (2), pólipos hiperplásicos en el recto de $10 \mathrm{~mm}$ y $4 \mathrm{~mm}$ (1), adenomas tubulares $<5 \mathrm{~mm}$ sin displasia (4), adenomas tubulares 
Tabla 2. Características sociodemográficas de la muestra poblacional

\begin{tabular}{|c|c|c|c|c|c|c|}
\hline & $\begin{array}{l}\text { Veracruz, } \\
\text { Veracruz }\end{array}$ & $\begin{array}{c}\text { Tuxtla Gutiérrez, } \\
\text { Chiapas }\end{array}$ & $\begin{array}{l}\text { Ciudad de } \\
\text { México }\end{array}$ & $\begin{array}{l}\text { Mérida, } \\
\text { Yucatán }\end{array}$ & $\begin{array}{l}\text { Puebla, } \\
\text { Puebla }\end{array}$ & Total \\
\hline$N(\%)$ & $430(57 \%)$ & $133(19 \%)$ & $118(17 \%)$ & $48(6 \%)$ & $22(3 \%)$ & $751(100 \%)$ \\
\hline Edad, años (DE) & $59.9(5.2)$ & $56.3(4.4)$ & $57.9(9.3)$ & $55.2(5.5)$ & $56.1(4.3)$ & $57.0(5.7)$ \\
\hline Hombres & 140 & 50 & 80 & 22 & 12 & 304 \\
\hline Ratio hombre:mujer & 0.48 & 0.6 & 2.1 & 0.84 & 1.2 & 0.68 \\
\hline Índice de masa corporal, kg/m² & 28.2 & 27.0 & 26.4 & 27.2 & 26.3 & 27.0 \\
\hline
\end{tabular}

DE: desviación estándar.

Tabla 3. Promedio de hemoglobina en heces $(\mathrm{ng} / \mathrm{ml})$ en la prueba inmunoquímica de sangre oculta en heces (FIT)

\begin{tabular}{|c|c|c|c|c|c|}
\hline \multirow{2}{*}{$\begin{array}{l}\text { Cololonoscopias } \\
\text { Total }\end{array}$} & \multicolumn{4}{|c|}{ FIT positivas } & \multirow[t]{2}{*}{$p$} \\
\hline & Total & Sin lesiones colorrectales & Lesiones no neoplásicas & Lesiones neoplásicas & \\
\hline N & 40 & 5 & 20 & 15 & \\
\hline Porcentaje & 100 & 12.5 & 50 & 37.5 & \\
\hline Hemoglobina (ng/ml) & 842 & 245 & 609.9 & 1350,2 & 0.014 \\
\hline Desviación estándar & 916 & 213 & 604.9 & 1186.6 & \\
\hline
\end{tabular}

Tabla 4. Comparaciones entre las cifras de hemoglobina en las heces de acuerdo con los hallazgos histopatológicos

\begin{tabular}{lc}
\hline Hallazgos & P (U de Mann Whitney) \\
\hline Negativos vs. positivos & 0.027 \\
Negativos vs. lesiones neoplásicas & 0.001 \\
Lesiones neoplásicas vs. no neoplásicas & 0.014 \\
\hline
\end{tabular}

con displasia leve (4), adenomas túbulo-vellosos con displasia leve (2), adenoma serrado (1) y adenocarcinoma invasor moderadamente diferenciado en el recto (1). En este último grupo de pacientes se reportaron también hallazgos no neoplásicos, como divertículos (2), pólipo hiperplásico (1), colitis ulcerosa crónica inespecífica (2) y fisura anal (1).

De las 751 pruebas procesadas, 51 (6.8\%) resultaron positivas, pero solo en 40 de estos pacientes se llevó a cabo una colonoscopia, con hallazgo de lesiones premalignas en 11 de ellos, lo que corresponde al $1.4 \%$ del total de los sujetos incluidos en el estudio, y hubo un paciente con adenocarcinoma invasor $(0.13 \%)$. Se calculó una tasa de 15.9 pacientes con lesiones neoplásicas con potencial de malignidad y cáncer por cada 1,000 sujetos evaluados, y de 1.3 pacientes con cáncer colorrectal por cada 1,000 sujetos evaluados, de acuerdo con la metodología de estudios previos.

Respecto a la comparación de la cuantificación de la $\mathrm{Hb}$ entre los grupos se reportó una diferencia estadísticamente significativa entre los pacientes con FIT positiva sin hallazgos de lesiones colorrectales por colonoscopia y aquellos con FIT positiva en los que sí hubo hallazgos por colonoscopia, tanto neoplásicos como no neoplásicos $(p=0.027, U$ de Mann-Whitney). De igual forma, al comparar las cifras $\mathrm{de} \mathrm{Hb}$ en los pacientes sin hallazgos endoscópicos y en los pacientes con hallazgos únicamente neoplásicos se evidenció una significancia estadística aún mayor ( $p=0.001, U$ de Mann-Whitney). Se demostró una diferencia significativa al comparar las cifras de $\mathrm{Hb}$ en los pacientes con hallazgos neoplásicos contra las de aquellos con hallazgos no neoplásicos $(p=0.014$, $U$ de Mann-Whitney) (Tablas 3 y 4).

\section{Discusión}

Actualmente existe suficiente evidencia en todo el mundo para afirmar que la FIT es más sensible y específica para la detección de lesiones neoplásicas colorrectales que la prueba de guayaco, y además 
Tabla 5. Comparación de resultados entre países según la literatura mundial

\begin{tabular}{|c|c|c|c|c|}
\hline & Uruguay $1996^{19}$ & Uruguay $2006^{20}$ & Tailandia $2013^{13}$ & México 2015 \\
\hline \multicolumn{5}{|l|}{ FIT } \\
\hline Número total & 1000 & 60,405 & 127,301 & 900 \\
\hline Procesadas & $805(8.5 \%)$ & $56,262(93.1 \%)$ & $80,012(62.9 \%)$ & $751(83.4 \%)$ \\
\hline Positivas & $117(14.6 \%)$ & $5299(9.4 \%)$ & $873(1.1 \%)$ & $51(6.8 \%)$ \\
\hline Colonoscopias & 70 & 3234 & 627 & 40 \\
\hline Número de lesiones neoplásicas & $48(68.5 \%)$ & $1266(39.1 \%)$ & $188(29.8 \%)$ & $15(37.5 \%)$ \\
\hline Número de casos de cáncer* & No reportado & $306(9.4 \%)$ & $23(3.7 \%)$ & $1(2.5 \%)$ \\
\hline Detección de neoplasia avanzada por cada 1000 casos* & No registrado & 14.6 & 28.7 & 1.3 \\
\hline
\end{tabular}

te

favorece una mayor adherencia a los programas de escrutinio por su practicidad, costo-beneficio y no invasividad $^{13}$. En este estudio, por primera vez en México demostramos que debería utilizarse esta prueba en población abierta en todo el país, ya que encontramos que el $6.8 \%$ de las pruebas son positivas y que el $1.4 \%$ y el $0.13 \%$ de nuestra población podrían tener lesiones premalignas o adenocarcinoma invasor, respectivamente.

En 1996 se llevó a cabo en Uruguay un estudio piloto realizando el primer escrutinio con FIT en población asintomática en América Latina, modelo en el cual nos basamos para el desarrollo del presente estudio $^{19}$. Incluyeron de forma prospectiva 1,000 sujetos usuarios del servicio de salud pública de Uruguay, asintomáticos, mayores de 50 años y $\sin$ antecedentes personales ni familiares de CCR (riesgo promedio). El $80.5 \%$ de los participantes (805) completaron el estudio y, de ellos, el $14.6 \%$ (117) fueron positivos, por lo que se les indicó una colonoscopia, que se realizó en 70 pacientes. Se encontraron 75 lesiones colorrectales en 48 pacientes, correspondiendo más de la mitad a pólipos, 28 de ellos adenomas. Posteriormente, en el año 2006, el grupo de investigación uruguayo publicó los resultados de un programa nacional de escrutinio con una muestra de 60,405 sujetos $^{20}$. La comparación de los resultados obtenidos en los diferentes estudios se muestra en la tabla 5.

El porcentaje de pacientes con resultado positivo en la FIT y hallazgo endoscópico de lesión neoplásica fue del $37.5 \%$ en nuestro estudio, en contraste con el $35 \%$ publicado en 2015 en el primer escrutinio efectuado en México mediante colonoscopia como único método de cribado ${ }^{21}$. Sin embargo, en todo el mundo esta cifra varía ampliamente desde el $16 \%$ hasta el $43 \%$, según los diferentes estudios. De igual forma, la tasa de detección de neoplasia avanzada por cada 1,000 sujetos evaluados oscila entre $1.1 \mathrm{y}$ $21^{15,16,22}$; en el presente estudio fue de 1.3 por cada 1,000 sujetos.

Si bien el estudio de García-Osogobio, et al. ${ }^{21}$ fue el primero sobre un programa de tamizaje para CCR reportado en el país, cabe señalar que se realizó en un tercer nivel de atención en los empleados de un solo centro privado en la Ciudad de México y con una muestra pequeña $(n=123)$. Nuestro estudio representa el primer esfuerzo multicéntrico en diversas partes del país y dirigido realmente a población abierta, la cual representa el objetivo primario de los programas nacionales de escrutinio para cualquier neoplasia.

El punto de corte utilizado en nuestro estudio fue de $100 \mathrm{ng} / \mathrm{ml}$, medida basada en la concentración de $\mathrm{Hb}$ en el líquido tampón, al igual que en el estudio realizado en Uruguay. Sin embargo, los consensos internacionales recomiendan la estandarización de la unidad de medida a microgramos por gramo $(\mu \mathrm{g} / \mathrm{g})$, es decir, la cantidad de $\mathrm{Hb}$ por cada gramo de heces $^{22,23}$.

En diversos estudios realizados en todo el mundo se ha demostrado que el promedio de cuantificación de la $\mathrm{Hb}$ aumenta de acuerdo con el grado de malignidad de la lesión. Por ejemplo, Fenocchi, et al. ${ }^{24}$ reportaron los siguientes promedios de $\mathrm{Hb}$ según el tipo de lesión: para adenoma $320 \mathrm{ng} / \mathrm{ml}$, para adenoma avanzado $608 \mathrm{ng} / \mathrm{ml}$, para cáncer precoz $730 \mathrm{ng} / \mathrm{ml}$ y para cáncer avanzado $931 \mathrm{ng} / \mathrm{ml}$. En nuestro estudio no fue posible identificar esta correlación, probablemente por el pequeño tamaño de la muestra total en comparación con otros estudios y la cantidad de lesiones halladas (solo un paciente con cáncer). Sin embargo, se demostró una diferencia estadísticamente significativa al comparar las cifras de $\mathrm{Hb}$ en los pacientes con hallazgos neoplásicos 
contra las de aquellos con hallazgos no neoplásicos ( $p=0.014$, U de Mann-Whitney).

La sensibilidad, la especificidad y los valores predictivos positivo y negativo de la FIT para la detección de CCR han sido reportados como el $100 \%$, el $90 \%$, el $16 \%$ y el $100 \%$, respectivamente, y para todas las neoplasias como el $74 \%$, el $93 \%$, el $45 \%$ y el $98 \%$, respectivamente. Una determinación fecal puede detectar el $100 \%$ de los CCR y el $74 \%$ de las neoplasias colorrectales significativas ${ }^{25}$. Por otra parte, unas tasas de positividad de la FIT de entre el $3 \%$ y el $5.9 \%$ son aceptables para realizar un escrutinio poblacional26,27. En Japón, 6 millones de personas (el $17 \%$ de la población elegible) han sido sometidas a la prueba $^{27}$.

En los países desarrollados (Estados Unidos de América y Canadá, Australia, Nueva Zelanda y la mayoría de los países europeos), la incidencia y la mortalidad del CCR han disminuido o se han estabilizado desde la década de 1970 debido a la reducción de la exposición a los factores de riesgo, la detección y la prevención tempranas, y la mejora de los tratamientos, a diferencia de lo ocurrido en los países en transición hacia niveles más altos de desarrollo humano, como los de América Central y del Sur, y los de Europa del Este, donde el CCR ha aumentado ${ }^{3}$.

El CCR tiene una incidencia cercana al $5 \%$, por lo cual es considerado como un verdadero problema de salud pública. Los programas de escrutinio son herramientas importantes para controlar la incidencia del CCR. Sin embargo, para ser efectivos deberían ir dirigidos a la población apropiada. El objetivo es la detección de lesiones precancerosas y de CCR incipiente en una población asintomática sin antecedentes de cáncer ni de lesiones precancerosas ${ }^{28,29}$.

La OMS ha implementado programas de escrutinio del CCR en las políticas poblacionales. El cáncer de mama y el CCR son los únicos con suficiente evidencia de la eficacia de su cribado para prevenir la ocurrencia o la progresión mediante un tratamiento adecuado ${ }^{28,29}$.

La tasa de detección de CCR puede fluctuar entre 0.37 y 0.45 dependiendo del punto de corte de la $\mathrm{Hb}$ (50-225 ng/ml), y se ha reportado que el número necesario de colonoscopias para detectar un caso de cáncer es de 11.7 con el punto de corte de $100 \mathrm{ng} / \mathrm{ml}^{30}$.

$\mathrm{Si}$ bien nuestro estudio representa un primer gran esfuerzo, es importante mencionar algunas de sus limitaciones. A pesar de ser un un estudio multicéntrico, puede observarse una gran heterogeneidad en la realización de FIT y de colonoscopias, y existen algunos sesgos de referencia y de selección. Por otro lado, es importante destacar que al realizar la colonoscopia también hay múltiples factores que pueden ser difíciles de controlar, como el tipo y la preparación de la colonoscopia, la técnica y la experiencia del endoscopista o de los centros, e incluso el tipo de equipo utilizado. Aun así, la importancia de nuestro estudio reside en que los hallazgos son similares a los reportados en otros lugares, y en los sitios donde más se capturaron pacientes, Ciudad de México, Chiapas y Veracruz, donde las cifras de positividad y la tasa de detección de lesiones premalignas fueron muy similares.

\section{Conclusiones}

En términos generales, los resultados de nuestro estudio concuerdan con lo reportado en la literatura mundial, apoyando así la iniciativa de fomentar el establecimiento de un tamizaje formal dentro del sector de salud pública. Se requiere un seguimiento y ampliar el tamaño de la muestra con el fin de evaluar el impacto sobre la incidencia y la mortalidad a largo plazo.

\section{Conflicto de intereses}

José M. Remes-Troche es miembro de los consejos asesores de Takeda Pharmaceuticals, Alfa-Wasserman y Almirall, y ha sido ponente para Takeda Pharmaceuticals, Asofarma, Alfa-Wassermnan, Almirall y Astra-Zeneca.

\section{Financiamiento}

No hubo ningún financiamiento para la escritura de este documento.

\section{Responsabilidades éticas}

Protección de personas y animales. Los autores declaran que para esta investigación no se han realizado experimentos en seres humanos ni en animales.

Confidencialidad de los datos. Los autores declaran que han seguido los protocolos de su centro de trabajo sobre la publicación de datos de pacientes.

Derecho a la privacidad y consentimiento informado. Los autores han obtenido el consentimiento informado de los pacientes y/o sujetos referidos en el artículo. Este documento obra en poder del autor de correspondencia. 


\section{Bibliografía}

1. Jemal A, Center MM, DeSantis C, Ward EM. Global patterns of cancer incidence and mortality rates and trends. Cancer Epidemiol Biomarkers Prev. 2010;8:1893-907.

2. International Agency for Research on Cancer. GLOBOCAN, 2012. Disponible en: $h$ ttp://globocan.iarc.fr/Pages/fact sheets population.aspx

3. Ferlay J, Colombet M, Soerjomataram I, Mathers C, Parkin DM, Piñeros $M$, et al. Global and regional estimates of the incidence and mortality for 38 cancers: GLOBOCAN 2018. Lyon: International Agency for Research on Cancer/World Health Organization; 2018.

4. Bray F, Ferlay J, Soerjomataram I, Siegel R, Torre L, Jemal A. Global cancer statistics 2018: GLOBOCAN estimates of incidence and mortality worldwide for 36 cancers in 185 countries. Ca Cancer J Clin. 2018:68:394-424

5. Maule M, Merletti F. Cancer transition and priorities for cancer control. Lancet Oncol. 2012;13:745-6.

6. Vogelstein B, Fearon ER, Hamilton SR, Kern SE, Preisinger AC Leppert M, et al. Genetic alterations during colorectal-tumor development. N Engl J Med. 1988;319:525-32.

7. Muto $T$, Nagawa $H$, Watanabe $T$, Masaki $T$, Sawada $T$. Colorectal carcinogenesis: historical review. Dis Colon Rectum. 1997;40.S80-5.

8. Bond $\mathrm{JH}$. Fecal occult blood tests in occult gastrointestinal bleeding Semin Gastrointest Dis. 1999;10:48-52.

9. López-Köstner F, Kronberg U, Zárate AJ, Wielandt AM, Pinto E, Suazo C, et al. Programa de detección de neoplasias colorectales en población mayor de 50 años. Rev Med Chil. 2012;3:281-6.

10. Mandel JS, Church TR, Bond JH, Ederer F, Geisser MS, Mongin SJ, et al. The effect of fecal occult blood screening on the incidence of colorectal cancer. N Engl J Med. 2000;343:1603-7.

11. Hoff G, Grotmol T, Skovlund E, Bretthauer M; Norwegian Colorecta Cancer Prevention Study Group. Risk of colorectal cancer seven years after flexible sigmoidoscopy screening: randomised controlled trial. BMJ. 2009;338:b1846.

12. Atkin WS, Edwards R, Kralj-Hans I, Wooldrage K, Hart AR, Northover JM, et al. Once-only flexible sigmoidoscopy screening in prevention of colorectal cancer: a multicentre randomised controlled trial. Lancet 2010;375:1624-33.

13. Khuhaprema T, Sangrajrang S, Lalitwongsa S, Chokvanitphong V, Raunroadroong T, Ratanachu-Ek T, et al. Organised colorectal cancer screening in Lampang Province, Thailand: preliminary results from a pilot implementation programme. BMJ Open. 2014;4:e003671.

14. Allison JE, Fraser CG, Halloran SP, Young GP. Population screening for colorectal cancer means getting FIT: the past, present, and future of colorectal cancer screening using the fecal immunochemical test for hemoglobin (FIT). Gut Liver. 2014;8:117-30

15. Colorectal Cancer Screening in the Americas. Situation and Challenges. WHO. Disponible en: https://www.paho.org/hq/dmdocuments/2016/Colorectal-Cancer-Screening-Landscape-English.pdf
16. Acosta-Padilla AM, Ceniceros RA, Reyes-Moctezuma GA, Rodríguez-Montoya RB, Rodríguez-Ramírez SE, Sandoval-Mex AM, et al. Detección oportuna y diagnóstico de cáncer de colon y recto no hereditario en adultos en primero, segundo y tercer nivel de atención. Guía de referencia rápida. Catalogo maestro de GPC. CENETEC; 2008.

17. Eishi Y. Fecal occult blood tests and their pitfalls. JICA, F.Y.; 2000.

18. Schreuders EA, Ruco A Rabeneck L, Schoen RE, Sung JJ, Young GP, et al. Colorectal cancer screening: a global overview existing programs. Gut. 2015;64:1637-49.

19. Montano-Morgade DE, Fenocchi-Moneda ER, Martínez-Gómez L, Rondán-Olivera M, Olano-Gossweiler AC. Screening en cáncer colorectal. Primer estudio de población asintomática en Uruguay. Cir Urug. 2000;70:14-9.

20. Fenocchi E, Martínez L, Tolve J, Montano D, Rondán M, Parra-Blanco A, et al. Screening for colorectal cancer in Uruguay with an immunochemical faecal occult blood test. Eur J Cancer Prev. 2006;15:384-90.

21. García-Osogobio J, Téllez-Ávila FI, Méndez N, Uribe-Esquivel M. Results of the first program of colorectal cancer screening in Mexico. Endoscopia. 2015;27:59-63.

22. Fraser CG, Allison JE, Halloran SP, Young GP; Expert Working Group on Fecal Immunochemical Tests for Hemoglobin, Colorectal Cancer Screening Committee, World Endoscopy Organization. A proposal to standardize reporting units for fecal immunochemical tests for hemoglobin. J Natl Cancer Inst. 2012;104:810-4.

23. Van Rossum, van Rijn AF, Laheij RJF, van Oijen MG, Fockens $P$, Jansen JB, et al. Cutoff value determines the performance of a semi-quantitative inmunochemical faecal occult blood test in a colorectal cancer screening programme. Br J Cancer. 2009;101:1274-81.

24. Fenocchi $F$, Gaggero $P$, Rondan M, Lopez-Alvarenga JC, Sobrino-Cossio S, Lambert N, et al. Usefulness of the fecal immunochemical test in the detection of advanced adenomas in subjects at average risk for colorectal cancer. Endoscopia. 2015;27:64-8.

25. Levy Z, Rozen P, Hazazi R, Vilkin A, Waked A, Maoz E, et al. Can quantification of faecal occult blood predetermine the need for colonoscopy in patients at risk for non-syndromic familial colorectal cancer? Aliment Pharmacol Ther. 2006;24:1475-81.

26. Allison JE, Feldman R, Tekawa I. Hemoccult screening in detecting colorrectal neoplasm: sensitivity, specificity y predictive value. Ann Intern Med. 1990;112:328-33.

27. Morikawa T, Kato J, Yamaji Y, Wada Y, Mitsushima T, Shiratori Y. A comparison of the immunochemical fecal occult blood test and total colonoscopy in the asymptomatic population. Gastroenterology. 2005;129:422-8.

28. Miller AB. Implementation of colon cancer screening: techniques, costs, and barriers. Gastroenterol Clin N Am. 2008;37:83-95.

29. von Karsa L, Lignini TA, Patnick J, Lambert R, Sauvaget C. The dimensions of the CRC problem. Best Pract Res Clin Gastroenterol. 2010;24:381-96.

30. Van Rossum, van Rijn AF, Laheij RJF, van Oijen MGH, Fockens $P$, Jansen JBMJ, et al. Cutoff value determines the performance of a semi-quantitative inmunochemical faecal occult blood test in a colorectal cancer screening programme. British Journal of Cancer 2009;101(8):1274-81. 